\title{
2D/3D seismic survey optimization of a hybrid (seabed node/towed streamer) project in a challenging environment
}

Dave Ridyard', Damian Hite' ${ }^{1}$, David J. Monk¹, ${ }^{1}$ ACTeQ LLC

Copyright 2021, SBGf - Sociedade Brasileira de Geofísica

This paper was prepared for presentation during the $17^{\text {th }}$ International Congress of the Brazilian Geophysical Society held in Rio de Janeiro, Brazil, 16-19 August 2021.

Contents of this paper were reviewed by the Technical Committee of the $17^{\text {th }}$ International Congress of the Brazilian Geophysical Society and do not necessarily represent any position of the SBGf, its officers or members. Electronic reproduction or represent any position of the SBGf, its officers or members. Electronic reproduction or
storage of any part of this paper for commercial purposes without the written consent storage of any part of this paper for commercial pur
of the Brazilian Geophysical Society is prohibited.

\section{Abstract}

As the margins for E\&P success and failure get ever smaller, geophysical surveys play an increasingly critical role in the exploration and production enhancement workflow. Each project represents a significant investment, with many stakeholders having subtly different, and potentially conflicting objectives. Geoscientists would like data that is of sufficient quality to meet their objectives. Managers want data delivered as soon as possible. Stockholders want to ensure that data is acquired with the lowest possible cost. Society demands that environmental impact is minimized. Optimization of all these factors is the key to a successful project. In this paper, we illustrate how this multivariate optimization can be accomplished for a project comprising both 2D and 3D elements. The 3D portion of the project is acquired using a combination of towed streamer and seabed nodes deployed around a fixed obstruction. The entire project is constrained to a short data acquisition window as a result of seasonal weather conditions.

Simple route optimization is insufficient to deal with the real world, in which geophysical crews face hazards, both static (rigs, reefs etc.) and dynamic (pipelaying barges, ships, marine mammals, tides etc.). In the marine environment, data may be acquired around these hazards using both towed streamer or seabed nodes, or a combined (hybrid) technique.

In this paper we show how a preliminary survey plan can be created based on a-priori knowledge of the environment, and how the plan can be updated as unexpected conditions arise.

It should be noted that an optimized survey is not just the most cost-effective solution, it is almost always the solution having the lowest environmental impact, due to reduced sound emissions (less shots) and reduced IMO regulated emissions of $\mathrm{CO} 2, \mathrm{NOX}$ and SOX etc. associated with reduced vessel survey distance.

Increasingly, these techniques learned in E\&P applications are being applied to the other offshore industries, such as site surveying for wind farms, and it is important that knowledge is effectively transferred to offshore wind and other related industries.

\section{Introduction}

The notional project considered here is a typical E\&P project, including a regional $2 \mathrm{D}$ grid designed to infill a pre-existing grid, and tie to a recent discovery well. A small $3 D$ survey is also planned around the discovery. Due to the cost of mobilizing multiple vessels to this remote area, a novel approach to acquiring critical data around the well is proposed, in which a small grid of seabed nodes is placed around the platform, and shots from the streamer survey are recorded.

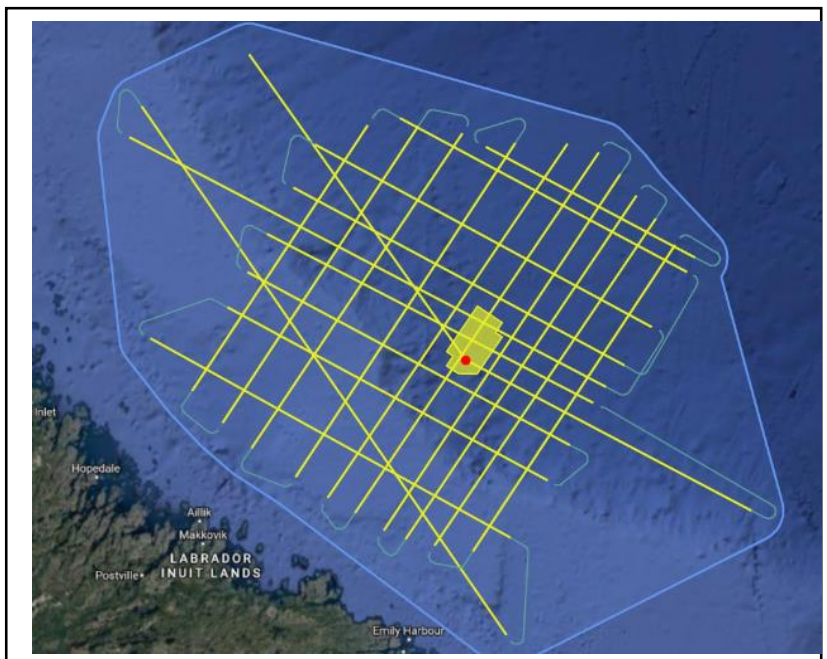

Figure 1 Proposed regional $2 D$ and targeted $3 D$ seismic surveys, shown with bathymetry and well location. Blue boundary indicates permitted operational region.

Many of the techniques discussed here are equally applicable to on shore surveys and time lapse projects (4D). Furthermore, we see that many of the techniques and challenges associated with geophysical surveys in E\&P applications are now increasingly being employed in other industries, such as wind farm construction. It is important that knowledge learned in E\&P applications is effectively transferred to the emerging offshore wind and other related industries.

We start by showing how a preliminary survey plan can be created based on a priori knowledge of the anticipated environment. We examine factors such as bathymetry, known obstructions in the area and predicted weather and currents. At this early stage we can estimate the cost of the project, and optimize the execution sequence. We can also the identify the best time of year to acquire the 
project based on modelled current predictions. Note that in practice, commercial, regulatory and environmental factors may force us to acquire data on a sub-optimal schedule.

For this project, the ideal data acquisition window extends from early June to early October. Outside of this window, adverse weather conditions can prove expensive, (Figure 2) and early in the season, icebegs can often be observed drifting southwards across the survey - their trajectories driven by prevailing currents and winds. (Figure 3 )

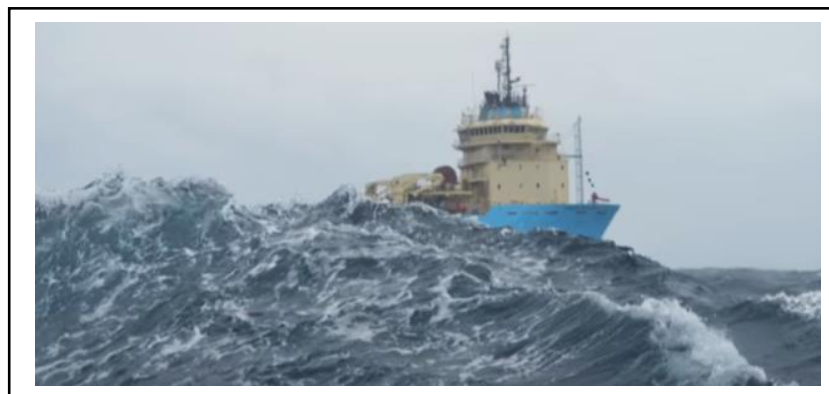

Fiqure 2 Tvpical October sea conditions

Optimization does not stop when the field crew commences work. The best pre-plan can only account for the known issues in the survey area. Inevitably, the real world will not match exactly the a priori knowledge available to the pre-planner. Airgun arrays will require maintenance. Uncooperative fishing vessels will force premature line changes. Once a deviation from the original pre-plan has occurred, the remaining portion of the survey still to be acquired should be re-optimized as if it was a new survey. This can be done offshore by the crew, or given reasonable remote internet access, this can be performed by onshore support personnel. For the case history considered here, we consider the impact of an iceberg drifting across the survey, but it could equally be a pipe-laying barge or a dive crew.

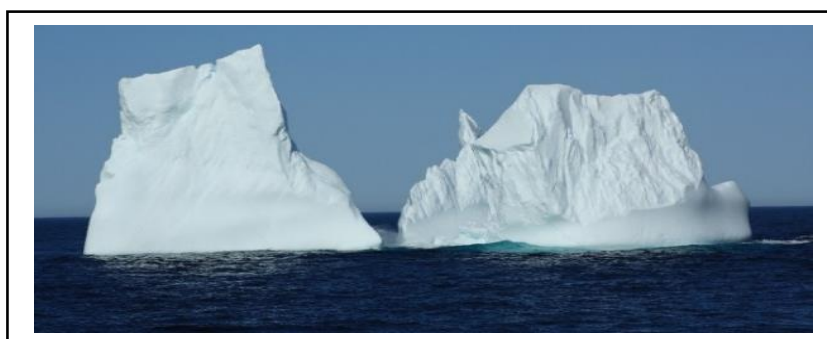

Figure 3 lcebergs are common in spring and early summer.

It should be noted that an optimized survey is not just the most cost-effective solution, it is almost always the solution having the lowest environmental impact. This is because if we can reduce the number of shots acquired, we reduce the total sound emissions. Furthermore, by reducing the total distance travelled by the survey vessel(s), we minimize IMO regulated emissions of $\mathrm{CO} 2$, NOX and SOX etc. associated with reduced vessel survey distance.

\section{D survey pre-plan}

Since a time consuming reconfiguration is necessary to convert the vessel from a $2 \mathrm{D}$ configuration to a $3 \mathrm{D}$ configuration (or vice versa) it was easy to see that the 2D survey and the 3D survey could be planned and optimized as discrete project elements. Figure 1 shows the optimized plan for the 2D survey. The 2D survey was estimated at 35 days of operations, including time to reconfigure the vessel from $3 D$ to $2 D$, and a downtime estimate based on prior experience. Note that another option would have been to avoid the reconfiguration cost by using separate $2 \mathrm{D}$ and $3 \mathrm{D}$ vessels. This option was swiftly dismissed as the extra mobilization cost significantly outweighed the time saving associated with the reconfiguration.

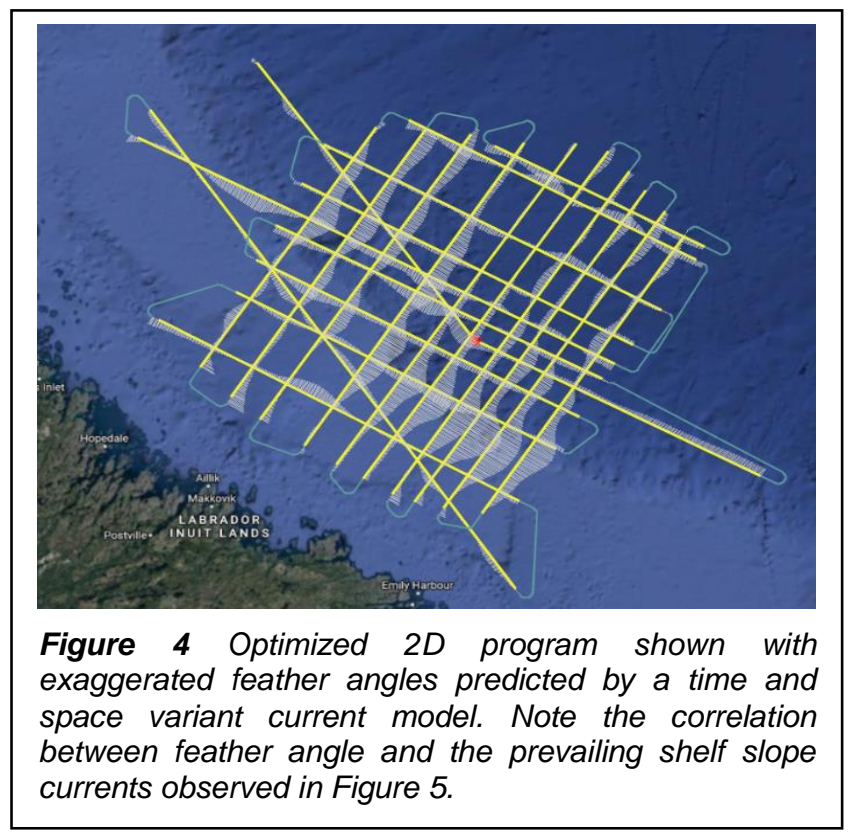

In designing the 2D program, consideration was given to ocean currents. Current data from prior years was examined, and currents were determined to follow fairly consistent seasonal and regional trends. These trends were notably influenced by the relatively steep seafloor slopes from the continental shelf into the deep ocean. Diurnal tidal variations were relatively insignificant in this area.

The dynamic current vectors were decomposed into the crossline component of current, which influences cable feathering (Figure 4) and the in-line component of current, which influences tow speed, strain on the towed streamer, time to acquire the survey, fuel consumption and total emissions.

\section{When should we acquire data?}

In this area, several hundred kilometres North of the sinking of the Titanic, the data acquisition window is severely constrained by weather conditions. Acquisition is only possible between May and September. May and June are prone to late breaking icebergs drifting southward across the survey area. 
A secondary factor in selecting the time of year to acquire the survey is that of cross currents. For a 3D seismic survey, towed streamers are deflected by any crossline currents. This is known as feathering. If the feathering angle on adjacent vessel passes is sufficiently different, then additional "in-fill" lines may need to be acquired. In some cases, this may be $30-50 \%$ of project time and cost. Consequently, we optimize the 3D acquisition by (1) Selecting the time of year with the lowest variation in cross currents (2) selecting the shooting sequence to minimize total acquisition time, including line change times and predicted infill.

Based on the weather and the current predictions shown in Figure 5, it was decided to acquire the 3D survey first, prior to the strong currents predicted for late July. The 2D survey would be acquired after the 3D, since cable feathering is considered to be less critical for 2D surveys.

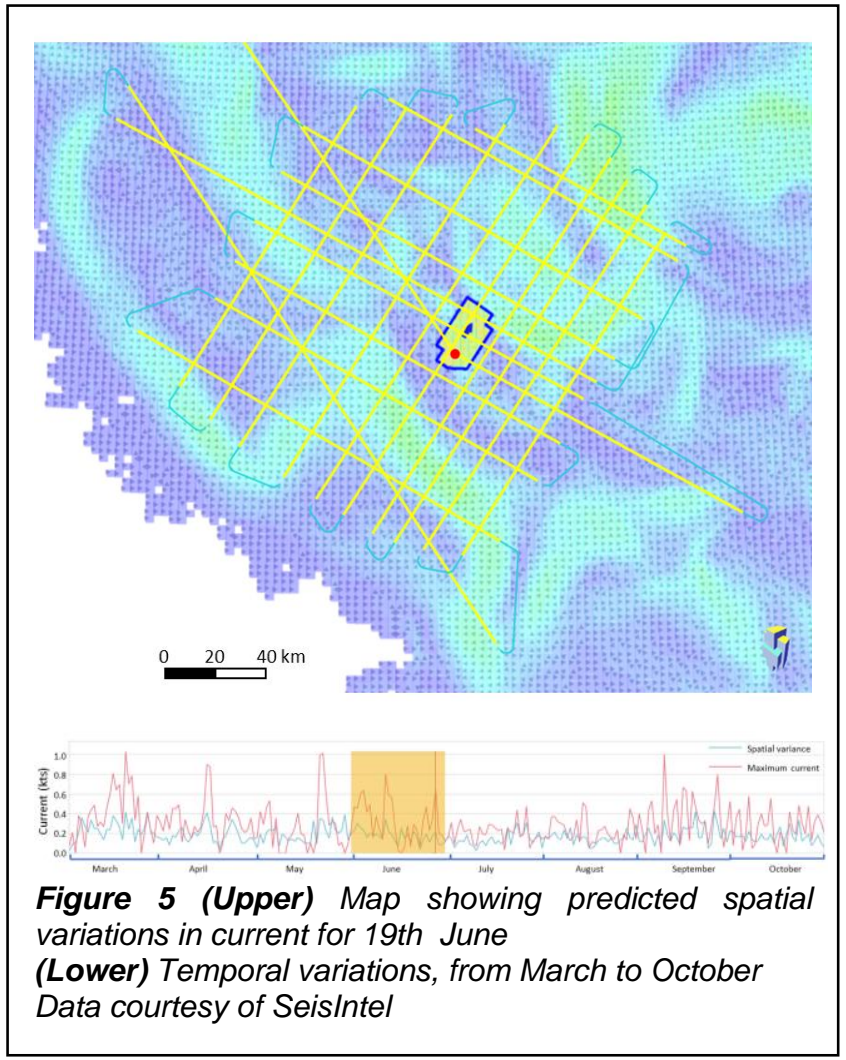

\section{D Survey Pre-Plan}

The final pre-survey planning step is to determine the acquisition sequence for the $3 D$ survey. Note that on this project a fixed production platform poses a challenge to towed streamer operations. Survey lines must be deviated around the platform to ensure safety of all field personnel and towed equipment. These deviations will cause a "hole" in the survey coverage. Traditionally these holes have either been ignored, leading to poor data quality or they have been undershot, using a second vessel, which can be prohibitively expensive. A third option is to shoot "deadhead" lines, in which the vessel is steered right up to the platform, and the line terminated abruptly. Deadheading reduces, but does not eliminate the coverage hole, and poses significant HSE concerns. What if the steering should fail right at the critical moment of closest approach to the platform?

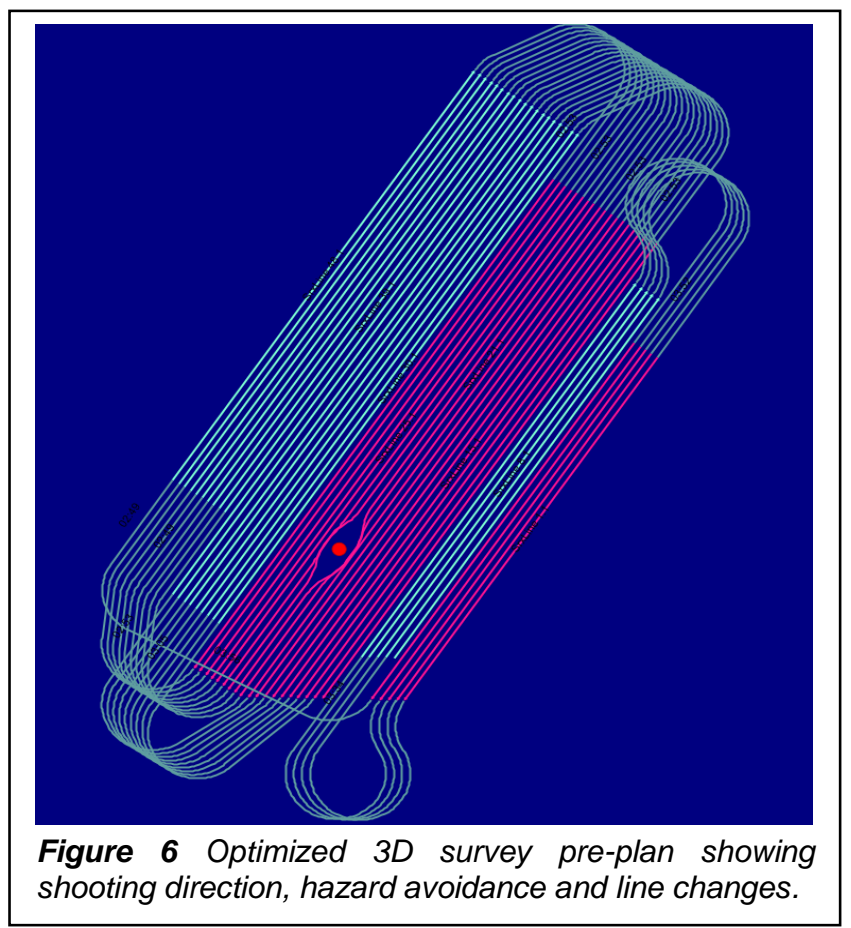

The recent arrival on the market of multi-purpose vessels (MPVs) capable of deploying seabed recording nodes and conducting conventional towed streamer acquisition has created another option for acquiring data under the platform. We elected to deploy 207 nodes around the platform, and record data from the streamer survey shots simultaneously on these nodes. The nodes add additional coverage, most notably, they improve coverage of vital short offset data, required for shallow imaging. (Figure 7) Deployment and recovery of the nodes added approximately 2 days to the total acquisition time. Note that this is less than the extra time required for other traditional solutions such as "deadheading", and it delivers far better continuity of coverage.

Optimizing 3D infill is a complex topic beyond the scope of this paper, but is more fully described by Hite ${ }^{1}$ et al. The estimated time to acquire the 3D survey, including predicted infill shooting is 27 days, with an additional 2 days to deploy and recover the seabed nodes. This results in an estimated total acquisition time of 64 days, including both 2D and 3D elements of the project.

\section{Field operations}

After completing about $10 \%$ of the survey, it became apparent that a large iceberg was drifting slowly to the south on a trajectory that would cross the North East corner of the survey. This required us to re-optimize the remainder of the project based on the new constraint imposed by this time variant obstruction (TVO). 


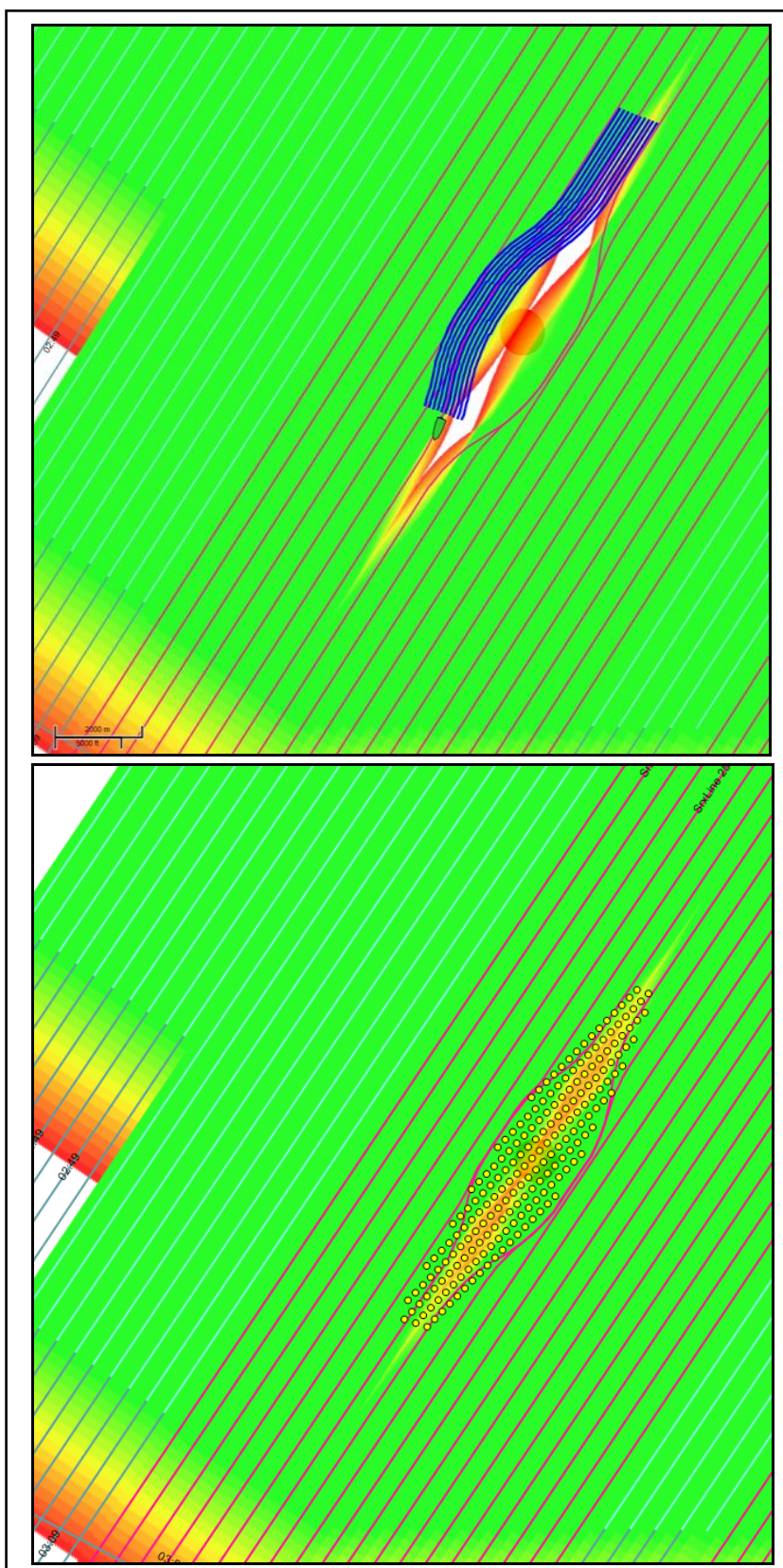

Figure 7 Preliminary 3D plan, prior to consideration of ocean currents and tides

(upper) coverage quality, or fold around the production platform with only streamer data. Note the streamer shape, allows "self-undershooting". As the vessel and streamers pass around the obstruction, some long offset data is recorded under the obstruction.

(lower) coverage quality with streamer plus node data. Node locations are shown as yellow circles.

After re-optimization, the iceberg added less than 4 hours to the acquisition time. (Figure 8)
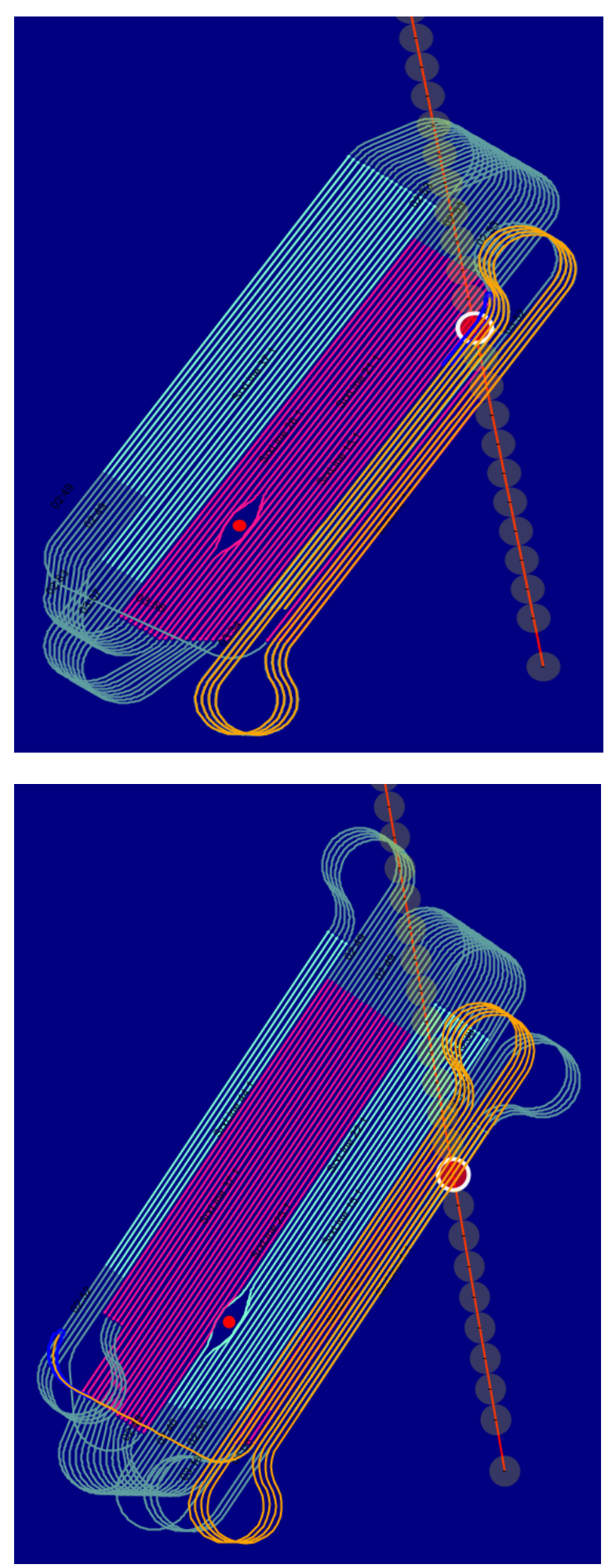

Figure 8 (upper) Original preplant, indicating potential intersection with iceberg.

(lower) Revised 3D plan - Adapted to maintain safe distance from southward drifting iceberg. 


\section{Conclusions}

Rigorous pre-survey planning, can significantly reduce

- overall project time, cost and uncertainty

- ensure adequate imaging quality

- personnel HSE exposure through reduced survey time

- environmental impact

- less shots means reduced total sound emissions to marine mammals

- reduced survey travel distance, reduces IMO regulated emissions of $\mathrm{CO} 2$, NOX, SOX etc.

An optimized pre-plan must be reoptimized as field conditions force adaptation to the initial plan.

Experience gained in oil and gas E\&P can be invaluable for other applications such as windfarm installation and renewable energy.

\section{References}

1) Optimizing Marine Data Acquisition and Infill, Damian A. Hite \& Dave Ridyard, ACTeQ, $2^{\text {nd }}$ EAGE Marine Acquisition workshop 25-26 August 2020 